\title{
Studying spike trains using a van Rossum metric with a synapse-like filter
}

\author{
Conor Houghton* \\ School of Mathematics, Trinity College Dublin, Ireland
}

6 May, 2008

\begin{abstract}
Spike trains are unreliable. For example, in the primary sensory areas, spike patterns and precise spike times will vary between responses to the same stimulus. Nonetheless, information about sensory inputs is communicated in the form of spike trains. A challenge in understanding spike trains is to assess the significance of individual spikes in encoding information. One approach is to define a spike train metric, allowing a distance to be calculated between pairs of spike trains. In a good metric, this distance will depend on the information the spike trains encode. This method has been used previously to calculate the timescale over which the precision of spike times is significant. Here, a new metric is constructed using a simple model of synaptic conductances. This metric proves effective at classifying neuronal responses by stimuli in the sample data set of electro-physiological recordings from the primary auditory area of the zebra finch fore-brain. The structure of the metric suggests that in these spike trains the significance of a spike is modulated by its proximity to previous spikes. This modulation is a putative information-coding property of spike trains.
\end{abstract}

*houghton@maths.tcd.ie 


\section{Introduction}

It is not known how the information that is propagating in the sensory pathways is encoded in spike trains. One approach to this question is to use a spike train metric (Victor and Purpura, 1996) to cluster responses to repeated presentations of a set of stimuli. If a metric measures a short distance between responses to the same stimulus and a longer distance between responses to different stimuli then the distance measured between two spike trains must be related to the information they encode. A metric can be evaluated by performing distance-based clustering and then calculating how accurately the metric clusters together responses to the same stimulus. Using this comparison to optimize a parameter in a family of metrics then gives a measurement of how information is coded in the spike trains.

This approach was used by Victor and Purpura (1996). This paper reports the analysis of spike trains collected from areas V1, V2 and V3 of awake monkeys during the transient, $256 \mathrm{~ms}$ long, presentation of visual stimuli which differ in texture and contrast level. The spiking responses were clustered using, what is now called, the Victor-Purpura metric: an edit-distance metric which has a free parameter $q$. An indicative time scale for the metric is given by $2 / q$; roughly speaking, spikes from two different spike trains can be thought of as being related by jitter if their timing difference is less than $2 / q$. It is a measure of the timescale for which spike timing is significant. In fact, the spike trains are clustered most accurately for $2 / q$ in the range c. $10-30 \mathrm{~ms}$ for stimuli which differed in contrast levels and c. $100 \mathrm{~ms}$ for stimuli which differed in texture. This shows that information is encoded in the spike timing, even though the individual visual stimuli are not time-dependent.

This same approach was applied to the neuronal responses of neurons in field L of zebra finches (Narayan et al., 2006; Wang et al., 2007). In the ascending auditory pathway, area field $\mathrm{L}$ is afferent to the song system and is considered the oscine analogue of the primary auditory cortex (Zaretsky and Konishi, 1976). However, different metric was used in this study. This study was similar to the one discussed above in that the metric had a timescale parameter and, again, optimizing this parameter showed that information is encoded in spike times.

The metric used in (Narayan et al., 2006; Wang et al., 2007) first converts a spike train into a function using a filter (Hunter et al., 1998; van Rossum, 2001). A metric on the space of functions then induces 
a distance measure on the space of spike trains. Provided the map between the space of spike trains and the function space is injective, as it is here, the induced distance measure will also be a metric.

To calculate the metric, the spike train is first filtered to form a function. The spike train, considered as a list of spike times, $\mathbf{t}=$ $\left(t_{1}, t_{2}, \cdots, t_{n}\right)$, is mapped to a real function, $f(t ; \mathbf{t})$ using a kernel $h(t)$ :

$$
\mathbf{t} \mapsto f(t ; \mathbf{t})=\sum_{i=1}^{n} h\left(t-t_{i}\right) .
$$

The distance between two spike trains, $\mathbf{t}_{1}$ and $\mathbf{t}_{2}$, is taken to be the distance between the two corresponding functions, using the standard $L^{2}$ metric on the space of real functions:

$$
d\left(\mathbf{t}_{1}, \mathbf{t}_{2}\right)=\sqrt{\int d t\left[f\left(t ; \mathbf{t}_{1}\right)-f\left(t ; \mathbf{t}_{2}\right)\right]^{2}} .
$$

One common choice of kernel is the decaying exponential

$$
h(t)=\left\{\begin{array}{ll}
0 & t<0 \\
e^{-t / \tau} & t \geq 0
\end{array} .\right.
$$

where $\tau$ is a time-scale which parameterizes the metric.

In Narayan et al. (2006) it was found that for zebra finch field $\mathrm{L}$ data, setting the time-scale $\tau$ to $12.8 \mathrm{~ms}$ gives the most accurate clustering of field L responses by song stimulus. Thus, $12.8 \mathrm{~ms}$ is an indicative time-scale for the encoding of information in these spike times.

In this paper, a number of different metrics will be compared. For convenience, the metric discussed above, based on a filter, will be refered to as the f-metric. The term van Rossum metric will be used to mean any metric induced on the space of spike trains by mapping individual spike trains to functions and then using a standard metric on the function space. Thus, the f-metric is a van Rossum metric. However, other van Rossum metrics will be considered which the map from spike train to functions is different.

Using stimulus-based clustering, it was found in both the examples discussed that information is carried by spike times. Furthermore, optimizing the metric timescale parameter gave an indicative timescale for the spike timing precision. Here, this investigation is continued. The f-metric is extended by changing the way the spike train is mapped 
to functions. The new filtering has an additional free parameter, one modelled on the short-term depletion of available binding sites in the dendritic spine. Varying this parameter improves the stimulus-based clustering of the zebra finch field L responses previously considered in Narayan et al. (2006). This indicates that this new van Rossum metric measures another property of information coding in the spike trains. Roughly speaking, the van Rossum timescale parameter measures the precision at which spike timing is significant. The new parameter measures how the significance of a spike varies depending on its proximity to previous spikes.

\section{Methods}

At the synapses, an arriving spike causes the exocytosis of vesicles containing neurotransmitters. The neurotransmitters diffuse across the synaptic cleft and binds with receptors on the dendritic spine. This triggers the opening of ion channels, changing the conductance of the dendritic membrane and leading to a post-synaptic potential. There is a rapid breakdown and reuptake of unbound neurotransmitters. The bound neurotransmitters detach from their binding sites, terminating the post-synaptic potential.

One simple way to model these conductance dynamics is to assume that the diffusion and binding of neurotransmitter is instantaneous and stereotypical. Thus, for conductance $g$,

$$
g \rightarrow g+\delta g
$$

whenever there is a pre-synaptic spike with $\delta g$ a constant. If the unbinding is assumed to be a constant rate stochastic process

$$
\tau_{s} \frac{d}{d t} g=-g
$$

with $\tau_{s}$ the constant synaptic time constant. Clearly, $g(t)$ can be identified with the filtered spike train $f(t ; \mathbf{t})$ used in the f-metric above. To make this more precise, $\tau_{s}$ is identified with $\tau$ and $f(t ; \mathbf{t})$ with $g / \delta g$.

A more complex model is now considered. This includes the depletion of available binding sites: some sites will already be occupied by neurotransmitters released by previous spikes. In this model, described in Dayan and Abbott (2001), the increase in conductivity is 
discontinuous but is not stereotypical: $g$ is modeled by

$$
g \rightarrow g+\frac{\delta g}{g_{\max }}\left(g_{\max }-g\right)
$$

for a pre-synaptic spike arriving and

$$
\tau_{s} \frac{d}{d t} g=-g
$$

to model stochastic unbinding. Thus, if $g$ is zero, it increases by $\delta g$ in response to a pre-synaptic spike. However, for non-zero $g$, the change is smaller and, if $g=g_{\max }$, there is no change.

Here, a new metric is defined by replacing the filtering of the spike train used in the van Rossum metric with a new map modeled on these synaptic dynamics:

$$
\mathbf{t} \rightarrow f(t ; \mathbf{t})
$$

where $f(t ; \mathbf{t})$ is the solution of

$$
\tau \frac{d}{d t} f=-f
$$

with discontinuities

$$
f \rightarrow(1-\mu) f+1
$$

at the spike times. Thus, $f(t ; \mathbf{t})$ is equivalent to $g / \delta g$ in the synaptic model and $\mu$ is equivalent to $\delta g / g_{\max }$. So, $\mu f$ can be thought of as the fraction of binding sites which are already occupied.

The distance between two spike trains $\mathbf{t}_{1}$ and $\mathbf{t}_{2}$ is now given by

$$
d\left(\mathbf{t}_{1}, \mathbf{t}_{2}\right)=\sqrt{\int d t\left[f\left(t ; \mathbf{t}_{1}\right)-f\left(t ; \mathbf{t}_{2}\right)\right]^{2}}
$$

with $0 \leq \mu \leq 1 . \mu=0$ corresponds to the original, f-metric with the exponential filter (3). For $\mu=1 f(t ; \mathbf{t})$ is reset to one at each spike. Since this metric is motivated by the depletion of binding sites, it will be refered to as the b-metric.

\section{Results}

This new metric has been applied to the electro-physiological data previously analyzed in Narayan et al. (2006) and in Wang et al. (2007). Narayan et al. (2006) should be consulted for a detailed description 
of the experimental and primary data processing procedures. The recordings were taken from field $\mathrm{L}$ of anaesthetized adult male zebra finches and data were collected from sites which showed enhanced activity during song playback. 24 sites are considered here. Of these, six are classified as single-unit sites and the rest as consisting of between two and five units (Narayan et al., 2006). The average spike rate during song playback is $15.1 \mathrm{~Hz}$ with a range across sites of 10.5$33 \mathrm{~Hz}$. At each site, ten responses to each of 20 zebra finch songs were recorded. In all the analyses here, one second of each recording is used, beginning at the onset of song playback. Example raster plots are presented in Fig. 1.

To examine the performance of the metrics we will follow the bootstrapped clustering procedure used in Victor and Purpura (1996). A confusion matrix, $N$, is calculated. This is a square matrix whose $i j$ th entry, $N_{i j}$, is the number of responses from stimulus $i$ which are closest, on average, to the responses from stimulus $j$. Given $n$ responses and $c$ stimuli, the responses are grouped into $c$ clusters according to their stimulus. Starting with a $c \times c$ matrix $N$ of zero entries, each response is considered in turn. It is temporarily removed and the average distance between it and the members of each of the $c$ clusters is calculated giving a set of average distances $\left\{d_{1}, d_{2}, \ldots, d_{c}\right\}$. If the smallest distance in this set is $d_{j}$ and the response being considered was taken from $i$ th cluster, one is added to $N_{i j}$. When all the responses have been considered, the elements of $N$ will add to $n$

$$
n=\sum_{i=1}^{c} \sum_{j=1}^{c} N_{i j} .
$$

In the case considered here, $c=20$ and $n=10 c=200$. As in Victor and Purpura (1996), the averaging over cluster elements is performed with a bias exponent. So, for a response $r$, the biased average distance to the $k$ th cluster is

$$
d_{k}=\left[\sum_{s \in C_{k}} d(r, s)^{z}\right]^{1 / z}
$$

where the bias is taken to be $z=-2$, under-weighting outliers.

If the metric is good at clustering the responses by stimulus, the confusion matrix will be largely diagonal. If it is poor, the off-diagonal entries will be approximately equal to the diagonal elements. As 
pointed out in Victor and Purpura (1996), the transmitted information, $h$, is a good quantitative measure of how well the responses are clustered. It is given by

$$
h=\frac{1}{n} \sum_{i j} N_{i j}\left(\ln N_{i j}-\ln \sum_{i} N_{i j}-\ln \sum_{j} N_{i j}+\ln n\right) .
$$

For perfect clustering, for $c$ equally likely stimuli, $h$ will be $\ln c$. For convenience, $\tilde{h}=h / \ln 20$ will be used here.

In Fig. 2, the performance of the synapse-based metric at different values of $\mu$ and $\tau$ is compared to the performance of the f-metric. Two comparisons are made. In Fig. 2A, metric parameters are optimized for each site, so, for each site the optimal performance of each metric is compared. In Fig. 2B, the same value of the parameter is used for all sites. Fig. 3 graphs the variation in $\tilde{h}$ with $\mu$ for fixed $\tau$.

In Fig. 2A, the optimal parameter values for each site are used for each of the two metrics. $\tilde{h}$ for the b-metric shows an average improvement of $14.5 \%$ over $\tilde{h}$ for the f-metric. For three sites, the improvement is over 30\%: the biggest improvement is $47 \%$. Most of the sites that showed little improvement have values of $\tilde{h}$ very close to one. This behaviour is not significantly different for the six sites which are classified as single neuron recordings: their average improvement is $12.0 \%$. The smaller value is likely to reflect the greater reliability of the single neuron sites. The average $\tilde{h}$ value is 0.868 using the f-metric whereas the average for all the sites is 0.802 .

The average parameter values are $\tau=12.9 \mathrm{~ms}$ and $\mu=0.72$ for the b-metric and $\tau=12.8 \mathrm{~ms}$ for the f-metric. In Fig. $2 \mathrm{~B}$, the performance is compared using these average values for all sites. Obviously, for most sites, the $\tilde{h}$ value is reduced for both metrics. The average improvement is also reduced slightly to $12.9 \%$. One site has a slightly lower $\tilde{h}$ value using the b-metric rather than the f-metric.

It is interesting to compare the performance of this b-metrics with other, similar, van Rossum metrics in which the map from spike train to function is defined to model different aspects of the conductance dynamics of synapses. One obvious modification is to take into account the finite rise time of the conductance as the neurotransmitters diffuse across the synaptic cleft and bind to their receptors. There is a simple model for this; a second, auxiliary, function $z(t ; \mathbf{t})$ is introduced, with

$$
\tau_{1} \frac{d f}{d t}=z-f
$$




$$
\tau_{2} \frac{d z}{d t}=-z
$$

with discontinuities

$$
z \rightarrow z+1
$$

at spike times. Roughly speaking, $\tau_{1}$ is the decay time, equivalent to the $\tau$ in the previous models and $\tau_{2}$ is the rise time. This reduces to the exponential filter function in the $\tau_{2} \rightarrow 0$ limit. If $\tau_{1}=\tau_{2}$, the response to a single spike will have the $t \exp \left(-t / \tau_{1}\right)$ alpha-function profile which is often used to describe synaptic responses. Like the bmetric discussed above, a van Rossum metric defined using this map from spike trains to functions gives a two-parameter generalization of the one-parameter f-metric. However the improvement in $\tilde{h}$ is much more modest. Comparing the optimal value of $\tau_{1}$ and $\tau_{2}$ at each site to the optimal f-metric gives an improvement of $3.3 \%$. Using average values of $\tau_{1}$ and $\tau_{2}$ and comparing to the f-metric using the average value of $\tau$ gives an improvement of just $2.0 \%$.

Synaptic depression is another important aspect of synaptic conductance dynamics. After a spike arrives, many synapses are observed to exhibit a reduction in efficiency with a timescale measured in hundereds of milliseconds. While this reduction is usually mild, the effect becomes significant in response to a burst of spikes. One mechanism for synaptic depression may be the depletion of the neurotransmittercarrying vescicles in the synapse. A simple model of synaptic depression was presented in Tsodyks and Markram (1997). Again, a second auxiliary function, $p(t ; \mathbf{t})$, is introduced and

$$
\begin{aligned}
\tau \frac{d f}{d t} & =-f \\
\tau_{d} \frac{d p}{d t} & =1-p
\end{aligned}
$$

with discontinuities

$$
\begin{aligned}
& f \rightarrow f+p \\
& p=\phi p .
\end{aligned}
$$

Thus, $f(t ; \mathbf{t})$ models a conductance with instantaneous binding and stochastic unbinding over a timescale $\tau . p(t ; \mathbf{t})$ is the number of available vesicles expressed as a proportion of the maximum possible vesicle number. $\phi \in[0,1]$ is a constant and $1-\phi$ is the fraction of available vesicles which are used in response to a single spike. $\tau_{d}$ is the timescale 
for recovery in vesicle numbers. The metric defined using this function will be refered to as the d-metric.

The biological situation motivating the d-metric is very different from the one motivating the b-metric discussed above. One models vesicle depletion, the other, the depletion of neurotransmitter binding sites. The time scales for these two biological phenomena are very different. Nonetheless, mathematically, the d-metric is a generalization of the b-metric. If $\tau_{d}=\tau$, then setting $p=1-\mu f$ and identifying $\phi$ with $1-\mu$ reduces the synaptic depression metric to the b-metric. For this reason, optimizing the three-parameter d-metric for each site will produce results which are superior to the b-metric. However, this improvement is modest: $2.9 \%$. Using average values, performance is actually degraded: the relative change is $-3.2 \%$. Synaptic facilitation has also been studied and does not appear to improve clustering performance for these data.

It seems that the performance gain exhibited by the b-metric is significant when compared to other biologically-motivated van Rossum metrics. In fact, the d-metric seems to only show superior performance because it over-fits the data. Of course, real synapses do not choose between binding-site depletion and synaptic depression; they have very complicated dynamics involving many different time scales. Nonetheless, it is interesting that modelling binding site depletion is particularly useful for clustering these data. These data are recorded from an area which is early in the auditory pathway where, perhaps, shorter timescales are particularly important. It is probable that the d-metric will demonstrate a larger improvement over the b-metric when applied to other data.

The f-metric and the Victor-Purpura metric (Victor and Purpura, 1996) have a similar clustering performance on the data considered here. However, the correlation-based similarity measure defined in Schreiber et al. (2003) is better. The correlation-based similarity measure, like the f-metric, is defined on the filtered spike-trains: $f(t, \mathbf{t})$. However, it uses a Gaussian filter

$$
h(t)=\frac{1}{\sqrt{2 \pi} \sigma} \exp \left(-\frac{t^{2}}{2 \sigma^{2}}\right)
$$

and the similarity $s\left(\mathbf{t}_{1}, \mathbf{t}_{2}\right)$ between two spike trains, $\mathbf{t}_{1}$ and $\mathbf{t}_{2}$ is the correlation of the two functions

$$
\left.s\left(\mathbf{t}_{1}, \mathbf{t}_{2}\right)\right)=\frac{\int f\left(t ; \mathbf{t}_{1}\right) f\left(t ; \mathbf{t}_{2}\right) d t}{\sqrt{\int f\left(t ; \mathbf{t}_{1}\right)^{2} d t \int f\left(t ; \mathbf{t}_{2}\right)^{2} d t}}
$$


Using optimal values of $\sigma$ and $\tau$, this similarity measure shows a $4.1 \%$ improvement over the f-metric. Using average values, the improvement is $4.7 \%$. What is interesting is that this similarity measure is quite different from the van Rossum metrics. Whereas the b-metric is physiologically motivated, the correlation-based similarity measure is motivated statistically. It seems that they are both superior to the f-metric when used to analyse these data. However, the reason that they perform better may be different in each case. This is evidence that there is further progress to be made in defining spike train metrics and in understanding how they capture salient features of spike trains.

\section{Discussion}

When used to cluster these data, the new metric shows an improvement over the original van Rossum metric. If this improvement holds for other data, the new metric has possible practical application for classifying neuronal responses. What is more striking,however, is the way the new metric depends on spike times: the significance of a spike depends upon its position relative to other spikes in the spike train. This is a novel putative feature of information coding in spike trains which can be measured by optimizing the parameter $\mu$. It appears to indicate that the precise pattern of spikes in a burst is less significant than the timing of isolated spikes. This is illustrated in Fig. 4 using examples of spike train clusters.

It is also interesting that this parameter is suggested by a simple model of synaptic conductance. Of course, the real dynamics of synapses are much more complicated and it would be interesting to understand binding site depletion, synaptic potentiation and depression and spike rate adaptation (Fairhall et al., 2001) as components in an information process.

\section{Acknowledgments}

C.J.H. was supported by a International Human Frontiers Science Program Organization short-term fellowship and by Science Foundation Ireland grant 06/RFP/BIM020. He is grateful to Kamal Sen for useful discussion and for the use of the data analysed here. I would like to thank one of the anonymous referees for suggesting Figure 4. 


\section{References}

Aronov D, Victor JD Non-euclidean properties of spike train metric spaces. Physical Review E, 69:061905, 2004.

Dayan P, Abbott LF. Theoretical Neuroscience. MIT Press, 2001.

Fairhall AL, Lewen GD, Bialek W, de Ruyter van Steveninck RR. Efficiency and ambiguity in an adaptive neural code. Nature, 412, 2001.

Hunter JD, Milton JG, Thomas PJ, Cowan JD. Resonance effect for neural spike time reliability. Journal of Neurophysiology, 80:14271438, 1998.

Narayan R, Graña G, Sen K. Distinct time scales in cortical discrimination of natural sounds in songbirds. Journal of Neurophysiology, 96:252-258, 2006.

H. Press WH, Teukolsky SA, Vetterling WT, Flannery, BP Numerical recipes 3rd Edition: the art of scientific computing Cambridge University Press, 2007.

Schreiber S, Fellous JM, Whitmer D, Tiesinga P, Sejnowski TJ. A new correlation-based measure of spike timing reliability. Neurocomputing, 52-54:925-931, 2003.

Tsodyks MV, Markram H. The neural code between neocortical pyramidal neurons depends on the neurotransmitter release probability. Proceedings of the National Academy of Science of the United States of America, 94: 719-723, 1997.

van Rossum M. A novel spike distance. Neural Computation, 13:751$763,2001$.

Victor JD, Purpura KP. Nature and precision of temporal coding in visual cortex: a metric-space analysis. Journal of Neurophysiology, 76(2):1310-1326, 1996.

Victor JD. Spike train metrics. Current Opinion in Neurobiology, 15(5), 2005. 
Wang L, Narayan R, Graña GM, Shamir M, Sen K. Cortical discrimination of complex natural stimuli: can single neurons match behavior? Journal of Neuroscience, 27(3):582-9, 2007.

Zaretsky, MD, Konishi, M Tonotopic organization in the avian telencephalon. Brain Research, 111:167-71, 1976. 
A

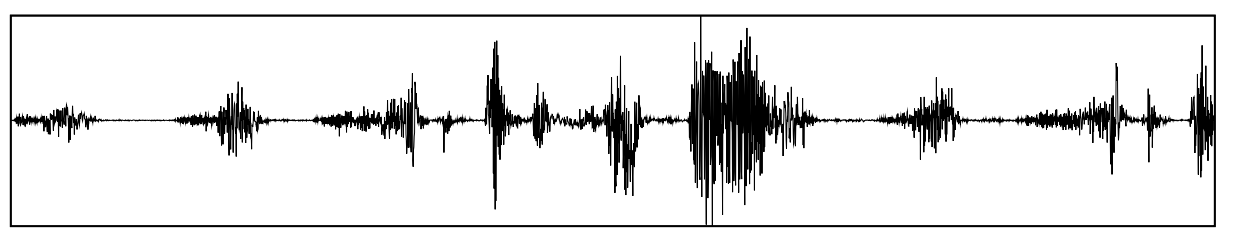

B

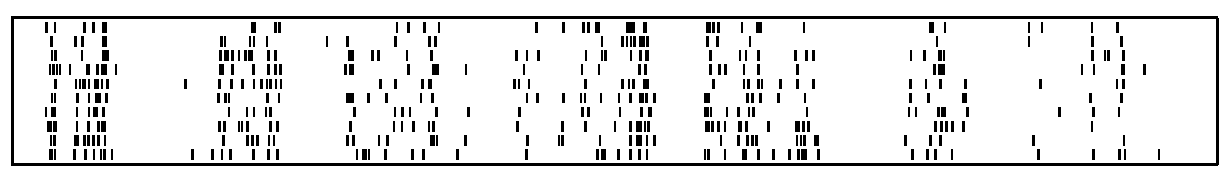

C

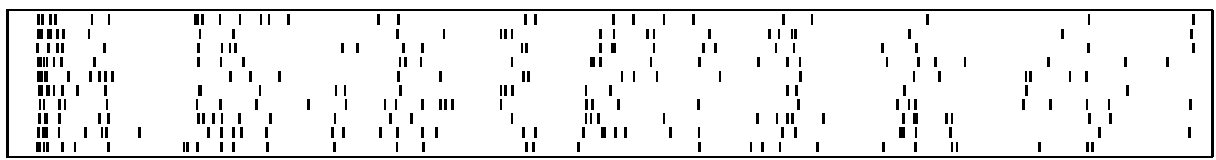

D

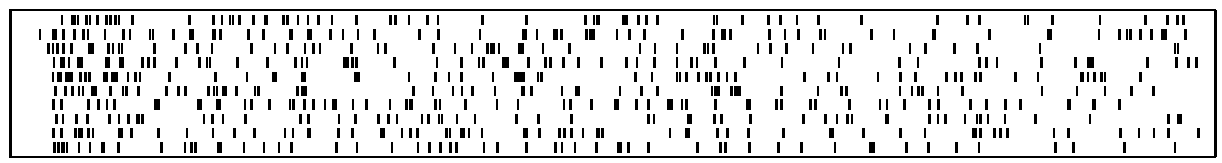

Figure 1: Examples of three raster plots. A shows the one second wave form of the stimulus song. In $\mathbf{B}, \mathbf{C}$ and $\mathbf{D}$ each box corresponds to the response at a single site to repeated stimulation with the song shown in $\mathbf{A}$. Time increases along the horizontal axis and the results of ten different trials are stacked vertically. A spike is marked by a vertical dash. Site $\mathbf{B}$ is unusually reliable. Using the measurement of reliability introduced in the Results section, $\tilde{h}=1$ calculated with both the f-metric and the b-metric. Site $\mathbf{C}$ is more typical with $\tilde{h}=0.91$ using the f-metric with $\tau=10 \mathrm{~ms}$ and $\tilde{h}=0.96$ using the b-metric with $\tau=17 \mathrm{~ms}$ and $\mu=1$. Site $\mathbf{D}$, in contrast, is among the worst sites. It shows very little coherence and poor performance on the cluster task. $\tilde{h}=0.36$ using the f-metric with $\tau=6 \mathrm{~ms}$ and $\tilde{h}=.43$ using the b-metric with $\tau=6 \mathrm{~ms}$ and $\mu=1.0$. 
A

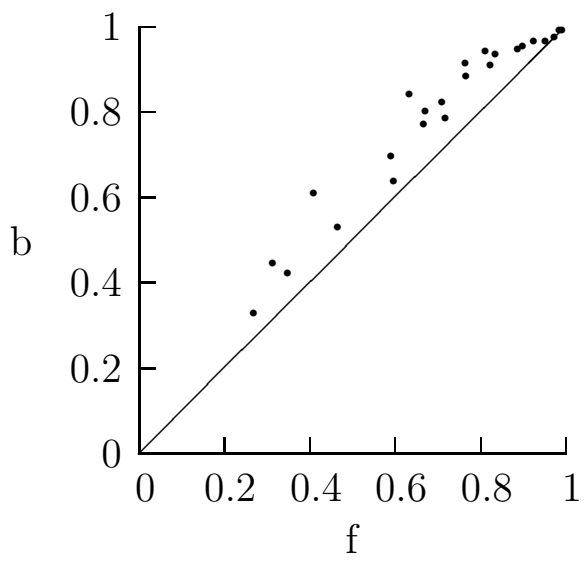

B

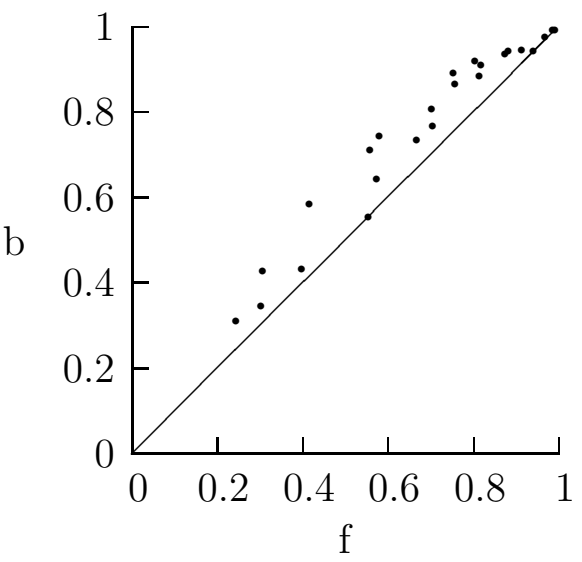

Figure 2: Comparing the b-metric to the f-metric. For each site, the parameter space is searched incrementally: $\tilde{h}$ values were calculated for $1 \mathrm{~ms} \leq \tau \leq 25$ $\mathrm{ms}$ at $0.5 \mathrm{~ms}$ intervals and for $0 \leq \mu \leq 1$ at intervals of 0.05 . This seems to be a sufficiently fine mesh, coarsening the mesh by a factor of two only changes the $\tilde{h}$ values in their fourth decimal place. This exhaustive search may seem a clumsy approach to maximizing $\tilde{h}$ and, since the $\tilde{h}$ landscape has, roughly, an inverted-bowl shape it might seem that this problem was ideally suited to numerical hill-climbing methods. However, the top of the inverted bowl has a very shallow ridge and because the data depends on the clustering of individual spike trains, the ridge profile is very grainy. Optimizing using the amoeba, amebsa and powell routines from Press et al. (2007) led to similar, but inferior, results. 


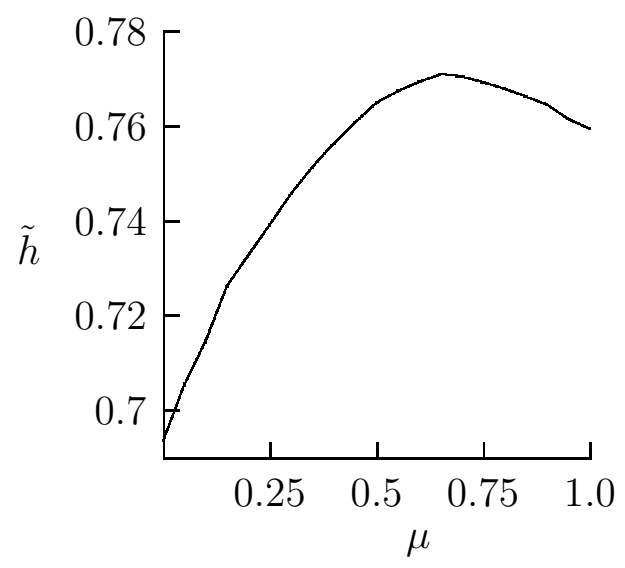

Figure 3: The variation in $\tilde{h}$ with $\mu$. $\tilde{h}$ has been calculated for all sites and averaged; $\tau$ is fixed at $\tau=12.875 \mathrm{~ms}$. 


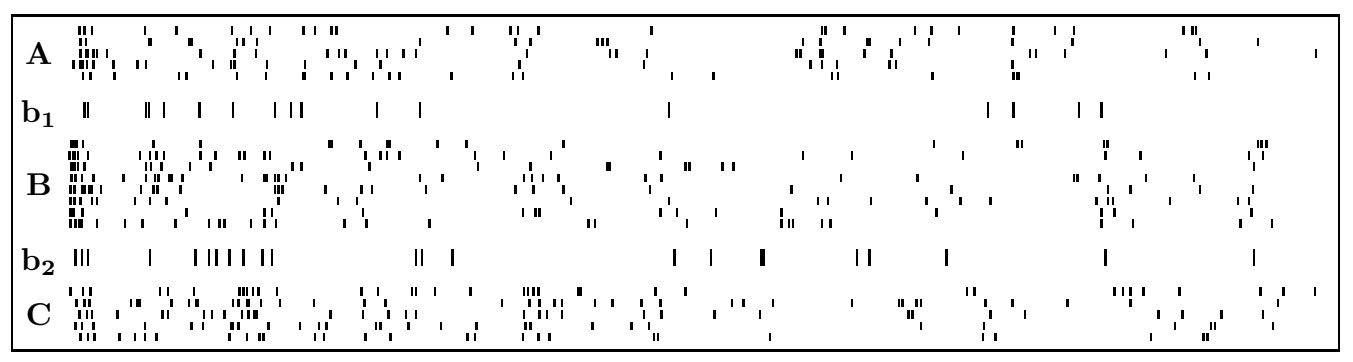

Figure 4: Spike trains which are misclassified under the f-metric but classified correctly under the b-metric. Here, rasters are plotted for one second of response by one site to three different songs. This site is the one which improves most in switching from the f-metric, with $\tilde{h}=0.42$, to the b-metric with $\tilde{h}=0.62$. Using this site may make it easier to interpret why the fmetric has a superior clustering performance. The rasters marked A, B and $\mathbf{C}$ are responses to different songs for which, in each case, the spike train is correctly classified by both the f-metric and the b-metric. The rasters marked $\mathbf{b}_{\mathbf{1}}$ and $\mathbf{b}_{\mathbf{2}}$ each show one response to song $\mathbf{B}$. The raster marks are double height for these responses, this is done for clarity and does not reflect any property of these spike trains. $\mathbf{b}_{\mathbf{1}}$ and $\mathbf{b}_{\mathbf{2}}$ are both incorrectly classified by the f-metric, $\mathbf{b}_{\mathbf{1}}$ is incorrectly assigned to $\mathbf{A}$ and $\mathbf{b}_{\mathbf{2}}$ to $\mathbf{B}$. Both spike trains are correctly assigned to $\mathbf{B}$ by the b-metric. This site shows a considerable degree of rate adaptation and it appears as if the f-metric gives undue weight to the spike times in the initial burst. The spike trains in $\mathbf{B}$ have a large number of spikes in this initial burst, something that is not matched by $\mathbf{b}_{\mathbf{1}}$ and $\mathbf{b}_{\mathbf{2}}$. However, the isolated spikes later in these spike trains more closely match those from responses to $B$. By reweighting the significance of these similarities, the b-metric correctly assigns $\mathbf{b}_{\mathbf{1}}$ and $\mathbf{b}_{\mathbf{2}}$. 